\title{
Investigation d'un cas suspect de Manifestations Adverses Post Immunisation (MAPI) dans le district sanitaire de la Commune 6, Bamako, mai 2021
}

\section{Investigation of a suspected case of Adverse Events Post Immunization of COVID-19 in the health district of Municipality 6, Bamako, may 2021}

Traoré T1a, Sangho $\mathrm{O}^{2,3^{*}}$, Konaké MDMS ${ }^{2 b}$, Traoré $\mathrm{M}^{2 c}$, Diabaté $\mathrm{ML}^{4}$, Dembelé $\mathrm{A}^{4}$, Sundika $\mathrm{T}^{5}$, Kokou $\mathrm{R}^{6}$, Sow $\mathrm{H}^{2}$, Diarra $\mathrm{B}^{2}$, Telly $\mathrm{N}^{3}$, Diallo $\mathrm{B}^{7}$, Coulibaly $\mathrm{CA}^{3,8}$, Kéïta $\mathrm{S}^{3}$, Berthé $\mathrm{M}^{8}$, Togola $\mathrm{OB}^{4}$, Kayembé $\mathrm{K}^{9}$, Traoré $\mathrm{B}^{9}$

\section{DOI : 10.53318/msp.v11i1.1903}

1 : Centre de Santé de Référence de la Commune 6 (CSRéf) du District de Bamako, Mali.

2 : Département d'Enseignement et de Recherche en Sciences Biologiques et Médicales (DERSBM), Faculté de Pharmacie (FAPH),

Université des Sciences, des Techniques et des Technologies de Bamako (USTTB), Bamako, Mali

3 : Département d'Enseignement et de Recherche en Santé Publique et Spécialités, Faculté de Médecine et d'Odonto-Stomatologie (FMOS)/USTTB, Mali

4 : Direction Générale de la Santé et de l'Hygiène Publique (DGSHP)

5 : AFRICA Center for Diseases Control and Preventions (CDC)

6 : Médecins Sans Frontière (MSF), Superviseur auprès du CSRéf

Commune 6, Bamako, Mali.

7 : Centre Hospitalier Universitaire, Centre National d'OdontoStomatologie (CNOS), Bamako, Mali

8 : Institut National de Santé Publique (INSP), Bamako, Mal

9 : African Field Epidemiology Network (AFENET-Mali), Bamako, Mali

*Auteur correspondant : Dr Oumar Sangho, Maître Assistant en Epidémiologie, Département d'Enseignement et de Recherche des Sciences Biologiques et Médicales (DERSBM), Faculté de Pharmacie (FAPH), USTTB, +223 762258 77, osangho75@gmail.com

a : a initié l'écriture de l'article sous la pulsion de l'auteur correspondant

b : est le Médecin Chef de District Sanitaire de la Commune 6 de Bamako.

c : est la responsable MAPI du District Sanitaire de la Commune 6 de Bamako qui a conduit la mission.

\section{Résumé}

Introduction : Comme tout vaccin, ceux contre la COVID19 peuvent provoquer des effets indésirables appelés Manifestations Adverses Post Immunisation (MAPI) ou manifestations post vaccinale indésirables. La MAPI ou manifestation post vaccinale indésirable est défini comme tout incident médical fâcheux qui suit la vaccination. L'objectif était d'investiguer un cas de MAPI avec décès. Méthodologie : C'était une étude descriptive qui s'est déroulée le 21 mai 2021 en Commune 6 du District de Bamako. Elle fait suite à la notification d'un cas de MAPI grave avec décès en Commune 6 de Bamako lors d'une consultation au cabinet médical Sorila. Résultats : II s'agissait du cas de Monsieur MR, âgé de 59 ans, avec des antécédents d'hypertension artérielle (HTA) non suivi ayant reçu une dose du vaccin Covishild de Astra Zeneca administrée par l'équipe mobile de vaccination à l'autogare de Sogoniko. II serait décédé dans la nuit du même jour. Son décès fut constaté à son arrivée au Cabinet médical Sorila au quartier Banakabougou en Commune 6 . Un prélèvement a été réalisé pour le diagnostic de la COVID-19, revenu négatif. Conclusion : Cette investigation nous a permis d'investiguer un suspect cas de MAPI grave avec décès dont la cause probable serait une maladie cardiovasculaire et de poser la nécessité d'une collaboration étroite entre structures publiques et privées.

Mots clés : Investigation, Manifestations Adverses Post Immunisation (MAPI), Décès, Bamako.

\begin{abstract}
Introduction: Like any vaccine, those against COVID-19 can cause side effects known as Adverse Post Immunization Events or adverse events following immunization (AEFI). AEFI or adverse event following vaccination is defined as any untoward medical incident that follows vaccination. The objective was to investigate a case of AEFI with death. Methodology: This was a descriptive study that took place on May 21, 2021 in Municipality 6 of Bamako. It follows the notification of a serious AEFI case with death in Municipality 6 of Bamako during a consultation at the Sorila clinic. Results: This was the case of Mr MR, 59 years old, with a history of unsuccessful hypertension who received a dose of the Covishild vaccine from Astra Zeneca administered by the mobile vaccination team at the terminal in Sogoniko. He would have died on the night of the same day. His death was pronounced on his arrival at the Sorila medical office in the Banakabougou district in Commune 6. A sample was taken for the diagnosis of COVID-19, with a negative income. Conclusion: This investigation allowed us to investigate a suspected case of serious AEFI with death whose probable cause would be cardiovascular disease and to pose the need for close collaboration between public and private structures.
\end{abstract}

Key words: Investigation, adverse events following immunization (AEFI), death, Bamako

\section{Introduction}

La maladie à coronavirus de 2019 (COVID-19) est causée par le coronavirus 2 du syndrome respiratoire aigu sévère (SRAS-CoV-2) (1). A la date du 3 mai 2021 le monde a enregistré au total 152.387.917 cas de COVID-19 rapportés dans les 223 pays et territoires touchés dans le monde (2). Parmi eux, on dénombre 3.195.624 décès, soit une létalité de 2,10\% (2). L'évolution journalière des données confirme la résurgence des cas de COVID-19 dans plusieurs pays comme c'est le cas actuellement en Inde avec en moyenne plus de 370.000 cas par jour et 3.500 décès au cours des 7 derniers jours (2).

En Afrique à la date du 3 mai 2021, au total 4.570 .906 cas confirmés y sont rapportés parmi lesquels 122.264 décès ont été enregistrés, soit une létalité de 2,7\% (2). Au Mali, 
le 9 mai 2021 la situation cumulée depuis le début de la maladie était 118.804 prélèvements analysés dont 14.108 cas positifs et 500 décès soit une létalité de $3,54 \%$, par ailleurs, nous avons noté 9.010 guéris, 841 en traitement dont 71 dans les établissements de santé et 770 à domicile, 8 transférés en dehors du pays (3).

Depuis son apparition les équipes de chercheurs à travers le monde ne cessent de s'activer pour trouver des remèdes en terme de soins curatifs et de soins préventif notamment à travers la vaccination $(1,4-7)$. Les vaccins ont été développés et déployés à une vitesse record. La facilité COVAX, un dispositif mené par le Global Alliance for Vaccine and Immunization (Gavi), par la Coalition pour les innovations en matière de préparation aux épidémies (CEPI) et l'Organisation Mondiale de la Santé (OMS), vise à accélérer le développement et la production de vaccins anti-COVID-19 et à garantir un accès juste et équitable à tous les pays du monde. (Lignes directrices OMS) (8). A la date du 15 avril, plus de 751,4 millions de doses de vaccins contre la COVID-19 ont été administrées à travers le monde (9).

Comme tout vaccin, ceux contre la COVID-19 peuvent provoquer des effets indésirables appelés Manifestations Adverses Post Immunisation (MAPI) ou manifestations post vaccinale indésirables (10). La MAPI est défini comme tout incident médical fâcheux qui suit la vaccination, n'a pas forcément un lien de causalité avec l'utilisation du vaccin, peut-être un symptôme défavorable dont se plaint une personne vaccinée, peut-être un résultat de laboratoire anormal, un symptôme ou une maladie constatée par un membre du personnel médical (10). Au 10 mars 2021, le nombre d'événements thromboemboliques signalés s'élève à 30 cas parmi environ 5 millions de personnes vaccinées avec le vaccin dans l'Union Européenne (UE) soit 0,0006 cas pour 100 individus, ce qui n'est pas plus élevé que le nombre observé dans la population générale (en l'absence du vaccin) (10).

Le 14 Mars 2021, le fabricant de Astra Zeneca a initié une revue des données concernant l'innocuité chez plus de 17 millions de personnes vaccinées dans l'UE et au Royaume-Uni (11). Le laboratoire affirme que 15 cas de thrombose veineuse profonde (TVP) et 22 cas d'embolie pulmonaire ont été signalés parmi les personnes vaccinées (11).

Au Mali, 69.933 personnes avaient reçu leur première dose à la date du 13 mai 2021 avec 9 cas de MAPI grave (12). Le 13 mai 2021, un cas de MAPI grave avec décès a été notifié en Commune 6 de Bamako lors d'une consultation au cabinet médical Sorila. L'objectif était d'investiguer le cas de décès. Le but était de circonscrire rapidement ce cas afin de minimiser son impact sur la poursuite normale de la campagne de vaccination.

\section{Méthodologie}

Cadre d'étude :

L'étude s'est déroulée dans le district sanitaire de la Commune 6 qui est couvert par un système de santé à deux échelons conformément à la politique sectorielle de santé :

$>$ Les Centres de Santé Communautaires (CSCom) au niveau des aires de santé constituent le premier échelon:

$>$ Le Centre de Santé de Référence (CSRéf) du district sanitaire (DS) représente le deuxième échelon.

La Commune dispose de 12 aires de santé (carte sanitaire 2021) au niveau du 1er échelon, dont les CSCom sont tous tenus par un personnel qualifié, avec au moins deux médecins et cinq sages-femmes chacun. Tous les CSCom sont impliqués dans la vaccination COVID-19.

Le CSRéf est le deuxième niveau du district sanitaire. II remplit essentiellement deux fonctions :

$>$ Une fonction de santé publique avec la coordination et la mise en œuvre d'activités de lutte contre la COVID-19. II dispose d'une équipe d'investigation.

$>$ Une fonction de référence clinique lui permettant de prendre en charge les cas de COVID-19 avec le dépistage, la communication des résultats, le suivi des contacts, la gestion du cordon aéroportuaire.

Une équipe pluridisciplinaire ou multisectorielle est impliquée dans la gestion de la COVID-19.

Type et période d'étude : il s'agissait d'une étude descriptive de cas qui s'est déroulée le 21 mai 2021.

Population d'étude :

Il s'agissait de la personne victime de MAPI.

Outils et technique de collecte des données :

Nous avons procédé à une revue documentaire des registres du Programme Elargi de Vaccination (PEV) au CSRéf et des registres de consultations au Cabinet Sorila et du CSRéf, à l'observation de la chaine de froid du PEV, à l'entretien avec les agents de santé du Cabinet médical Sorila et du CSRéf, à l'entretien avec les parents et amis du défunt.

Définition de cas de MAPI :

La MAPI ou manifestation post vaccinale indésirable est défini comme tout incident médical fâcheux qui Suit la vaccination, N'a pas forcément un lien de causalité avec l'utilisation du vaccin, Peut-être un symptôme défavorable dont se plaint une personne vaccinée, Peut-être un résultat de laboratoire anormal, un symptôme ou une maladie constatée par un membre du personnel médical. Traitement et analyse des données :

Les données collectées ont été présentées sous forme narrative.

Considérations éthiques :

La confidentialité et l'anonymat ont été garantis. Aucune donnée ne permettait d'identifier la victime. Les initiales des noms ont été utilisées et certains ont été changés afin de garantir l'anonymat.

\section{Résultats \\ Description du cas}

II s'agissait du cas de Monsieur AB, âgé de 59 ans, résidant au quartier Niamakoro dans l'aire de santé de ASACONIA en Commune 6 de Bamako. En date du 13 mai 2021, à 12 heures, il s'est présenté à l'équipe mobile 
de la vaccination contre la COVID-19 à l'autogare de Sogoniko pour recevoir sa première dose du vaccin Astra Zeneca. Après interrogatoire relatif aux antécédents médicaux, il a été noté qu'il avait une cardiopathie mais pas d'antécédent d'allergie connu.

Les formalités d'usages ayant été rempli, notamment l'enregistrement de toutes les informations dans les outils de renseignements tel que recommandé par les procédures relatives à la vaccination contre la COVID-19. Le vaccin administré est issu du lot $4121 Z 006$ nommé Covishild. La chaine de froid avait été respectée.

II avait reçu une injection de $0,5 \mathrm{ml}$ du vaccin Covishild de Astra Zeneca au niveau du deltoïde gauche et aucun retour de sang n'a été constaté après l'injection. Après quinze minutes d'observation, on n'a signalé aucune réaction particulière. Une carte de vaccination a été délivré avec tous les renseignements notamment le numéro de téléphone du point focal de pharmacovigilance et la date du prochain rendez - vous.

Le matin du 14 mai 2021 à 9 heures 44 minutes, le point focal de la pharmacovigilance a été appelé pour l'informer du décès de Monsieur $A B$. Une rencontre a été immédiatement initiée au CSRéf de la Commune 6 pour discuter dudit cas, regroupant le Médecin Chef et l'équipe du système d'information sanitaire qui ont décidés de mener une investigation sur ce cas de décès.

Une équipe a été envoyée sur le terrain au niveau de l'autogare de Sogoniko pour collecter les informations relatives aux circonstances du décès. Lors des entretiens l'atmosphère était tendue. II serait décédé dans la nuit à l'autogare. Conduit à la clinique Sorila au quartier Banakabougou en Commune 6, l'équipe de garde constatera son décès à l'arrivée.

A l'examen clinique, il y avait une mydriase bilatérale aréactive, une déviation labiale et un arrêt cardio respiratoire d'où la suspicion d'un accident vasculaire cérébral (AVC).

Un entretien approfondi a permis de noter les antécédents dont l'hypertension artérielle sur fond de mauvaise compliance du traitement et de doute quant à l'existence de la maladie.

Une visite au service du PEV du CSRéf a été effectuée pour vérifier la chaine de froid et s'entretenir avec les équipes de vaccination sur l'application des procédures relatives à la vaccination contre la COVID-19.

\section{Examen de laboratoire :}

Un prélèvement a été réalisé par l'équipe d'investigation. Il a été acheminé au laboratoire pour la confirmation de I'hypothèse diagnostic de COVID-19. Le résultat est revenu négatif.

Recherche active d'autres cas de MAPI :

A l'issu du dépouillement des registres de consultations au Cabinet Sorila et au CSRéf aucun cas de MAPI ou de décès n'a été détecté.

Identification du flacon du vaccin et des personnes ayant reçu les doses de ce flacon :
Les autres quatre personnes, ayant reçu les doses du même flacon, se portaient bien.

Actions de santé publique :

A l'issue de l'investigation, les actions suivantes ont été entreprises. II s'agit de :

- Mise en alerte des agents de santé ;

- Vérification et Diffusion des directives techniques sur les cas de MAPI ;

- Briefing des agents de santé sur les MAPI ;

- Planification des activités de sensibilisation dans l'entourage du défunt (autogare, famille)

- Recherche active d'autres cas dans la communauté et dans les structures de santé.

\section{Discussion :}

Au cours de cette étude, certaines difficultés ont été notées, pouvant constituer des limites dont:

- Manque de formation sur les procédures et directives techniques sur la vaccination contre la COVID-19 dans les structures privées,

- Insuffisance de collaboration entre les structures privées et les aires de santé sur les procédures relatives au système d'information sanitaire, Insuffisance de support de collecte des données et de kits de prélèvement dans le cabinet.

La vaccination est une des meilleurs moyens pour prévenir la maladie (13) et garantir un contrôle de cette pandémie $(14,15)$. Cependant, malgré toutes les précautions et toute l'organisation autour d'elle, elle peut comporter des risques surtout considérant les empressements pour trouver des vaccins $(5,16-18)$.

A la date du 15 avril, plus de 751,4 millions de doses de vaccins contre la Covid-19 ont été administrées à travers le monde (10).

L'Afrique est confrontée à des défis considérables pour garantir l'accès aux vaccins contre la COVID-19 et atteindre son objectif de vacciner jusqu'à $60 \%$ de la population sur le continent (11). Au 15 mars 2021, 23,6 millions de doses de vaccin ont été distribuées sur le continent africain, ce qui correspond à une couverture de seulement 1,7\% (11). Le vaccin Astra Zeneca, alloué principalement par le biais de l'initiative COVAX, représente 20,5 millions de ces doses constituant actuellement l'épine dorsale des campagnes de vaccination en Afrique (11).

Le nombre de personnes ayant reçues la première dose dans la Commune 6 à la date du 13 mai 2021 (37ème jour de vaccination) était de 19.287 personnes. Aucun autre cas de MAPI n'a été notifié dans la Commune (12). Par ailleurs, au plan national, il y avait 18 cas de MAPI notifiés dont 9 graves (thromboses veineuses) avec 6 cas de patients déjà positifs au SRAS COV2 au moment de la vaccination (12). Ces cas avaient tous moins de 30 ans sauf une patiente de 42 ans avec des antécédents de thrombose veineuse (12).

Des efforts ont été fait par l'OMS dans le cadre de l'assurance qualité de la vaccination notamment contre 
COVID-19 (13). Malgré ces efforts, des cas de MAPI graves ont été signalé dans certains pays dont : l'Autriche où le 7 mars 2021, l'Office Fédéral Autrichien pour la Sécurité dans les Soins de Santé (BASG) a signalé un décès survenu 10 jours après des troubles graves de la coagulation ainsi qu'un cas nécessitant une hospitalisation suite à une embolie pulmonaire ; le Danemark où le 11 mars 2021, l'Autorité Danoise de la Santé et des Médicaments a signalé un décès suite à des caillots sanguins ; la Norvège où le 14 mars 2021, l'Agence Norvégienne des médicaments a fait état d'un décès inattendu suite à une hémorragie cérébrale et de trois autres cas de caillots sanguins ou d'hémorragies cérébrales chez des personnes de moins de 50 ans nécessitant une hospitalisation ; les Pays Bas où le 14 mars 2021, le Centre Néerlandais de Pharmacovigilance (Lareb) a annoncé 10 potentiels cas ayant été signalés dans le pays (11).

L'analyse des données issues de l'investigation de ce cas ne sont pas suffisantes pour confirmer la MAPI liée à la vaccination COVID-19. Les moyens d'investigation technique dans ce sens étaient limités et la famille n'a pas acceptée une investigation clinique approfondie. Cela se comprend vu les considérations religieuses fasses aux dépouilles mortelles, surtout dans un pays à grande majorité musulmane.

\section{Conclusion}

Cette étude nous a permis d'investiguer un cas probable de MAPI grave avec décès chez un homme de 59 ans ayant des antécédents de maladie cardiovasculaire. Les moyens techniques disponibles n'ont pas permis de confirmer la MAPI suivant la vaccination contre la COVID19. Cependant, la notification de ce cas a été une alerte pour rappeler aux prestataires et à la communauté les mécanismes de gestion de MAPI dans le cadre de la campagne de vaccination contre la COVID-19. L'étude a par ailleurs posé la nécessité d'une collaboration étroite entre structures publiques et privées, de la sensibilisation de la communauté sur la vaccination contre la COVID-19 et de la notification systématique de tous les cas de MAPI

\section{Références}

1. Beeraka NM, Tulimilli SV, Karnik M, Sadhu SP, Pragada RR, Aliev G, et al. The Current Status and Challenges in the Development of Vaccines and Drugs against Severe Acute Respiratory Syndrome-Corona Virus-2 (SARS-CoV-2). BioMed Res Int. 1 juin $2021 ; 2021: 8160860$.

2. Centre des Opérations d'Urgences de Santé Publique. COVID-19, Bulletin d'information

hebdomadaire 11, Semaine du 26 avril au 02 mai 2021 [Internet]. Burundi: Ministère de la Santé Publique et de la lutte contre le Sida, Rép. du Burundi; 2021 mai p. 14. Report No.: 11. Disponible sur: https://www.afro.who.int/sites/default/files/202106/Burundi_Bulletin\%20Hebdomadaire\%20COVID19\%20Num\%C3\%A9ro\%2011.pdf
3. OCHA Services. Bulletin épidémiologique hebdomadaire $n^{\circ} 18$ (Du 03 au 09/05/2021) - Mali [Internet]. ReliefWeb. 2021 [cité 17 juill 2021]. Disponible sur: https://reliefweb.int/report/mali/bulletin-pidmiologique-hebdomadaire-n-18-du-03-au-09052021 4. Krumm ZA, Lloyd GM, Francis CP, Nasif LH, Mitchell DA, Golde TE, et al. Precision therapeutic targets for COVID-19. Virol J. 29 mars 2021;18:66. 5. Sharma A, Ahmad Farouk I, Lal SK. COVID-19: A Review on the Novel Coronavirus Disease Evolution, Transmission, Detection, Control and Prevention. Viruses. 29 janv 2021;13(2):202.

6 . De P, Chakraborty I, Karna B, Mazumder N. Brief review on repurposed drugs and vaccines for possible treatment of COVID-19. Eur J Pharmacol. 5 mai 2021;898:173977.

7. Won J-H, Lee H. The Current Status of Drug Repositioning and Vaccine Developments for the COVID19 Pandemic. Int J Mol Sci. 21 déc 2020;21(24):9775.

8. OMS. COVAX : collaboration en vue d'un accès mondial et équitable aux vaccins contre la COVID-19 [Internet]. OMS Genève; 2021 [cité 17 juill 2021]. Disponible sur: https://www.who.int/fr/initiatives/actaccelerator/covax

9. ONU. Covid-19 dans le monde : le nombre de nouveaux cas hebdomadaires a doublé en deux mois (OMS) [Internet]. ONU Info. 2021 [cité 17 juill 2021]. Disponible sur: https://news.un.org/fr/story/2021/04/1094062

10. OMS. Les effets indésirables des vaccins contre la COVID-19 [Internet]. OMS Genève; 2021 [cité 13 juill 2021]. Disponible sur:

https://www.who.int/fr/news-room/featurestories/detail/side-effects-of-covid-19-vaccines 11. Africa Union, Africa CDC. Communiqué aux États membres concernant les préoccupations liées au déploiement du vaccin COVID-19 d'AstraZeneca, en relation avec les rapports d'événements indésirables en Europe [Internet]. 2021 [cité 17 juin 2021]. Disponible sur: https://africacdc.org/wpcontent/uploads/2021/03/FACDC-AstraZeneccaStatement 22march-french.pdf

12. Ministère de la Santé et du Développement Social ; Direction Générale de la Santé et de l'Hygiène Publique ; Sous-Direction de Lutte contre la MaladieSection Immunisation. Résultats provisoires de la Campagne de vaccination contre la COVID-19 dans le District de Bamako à J37. Bamako, Mali: Ministère de la Santé et du Développement Social; 2021.

13. WHO. Vaccines and immunization: Vaccine safety [Internet]. WHO Geneva; 2020 [cité 13 juill 2021]. Disponible sur: https://www.who.int/news-room/q-adetail/vaccines-and-immunization-vaccine-safety 14. Jeyanathan M, Afkhami S, Smaill F, Miller MS, Lichty BD, Xing Z. Immunological considerations for COVID-19 vaccine strategies. Nat Rev Immunol. 4 sept 2020;1-18. 
15. Li Y-D, Chi W-Y, Su J-H, Ferrall L, Hung C-F, Wu T-C. Coronavirus vaccine development: from SARS and MERS to COVID-19. J Biomed Sci. 20 déc 2020;27:104.

16. Moore JP, Klasse PJ. COVID-19 Vaccines: "Warp Speed" Needs Mind Melds, Not Warped Minds. J Virol. 17 août 2020;94(17):e01083-20.

17. Poland GA, Ovsyannikova IG, Kennedy RB.

SARS-CoV-2 immunity: review and applications to phase
3 vaccine candidates. Lancet Lond Engl. 2020;396(10262):1595-606.

18. Pushparajah D, Jimenez S, Wong S, Alattas H, Nafissi N, Slavcev RA. Advances in gene-based vaccine platforms to address the COVID-19 pandemic. Adv Drug Deliv Rev. mars 2021;170:113-41. 